\title{
Bienvenida Mesa Directiva 2020-2022
}

\author{
Welcome Board 2020-2022
}

\author{
Federico Javier Juárez-de la Cruz, * Josefina Alberú-Gómez, ${ }^{\ddagger}$ Carmen Gracida Juárez ${ }^{\S}$ \\ * Editor en Jefe de la Revista Mexicana de Trasplantes. \\ ‡ Instituto Nacional de Ciencias Médicas y Nutrición «Salvador Zubirán». \\ $\S$ Hospital de Especialidades, Centro Médico Nacional Siglo XXI, IMSS.
}

En representación de la Mesa Directiva 2020-2022 de la Sociedad Mexicana de Trasplantes que preside el Dr. Rodrigo López Falcony y su Mesa Directiva, les doy la más cordial bienvenida a un nuevo periodo de gestión y que continúe en forma exitosa, tal como se ha logrado desde su fundación. Se inicia también un nuevo periodo con el cambio del Editor en Jefe de la Revista Mexicana de Trasplantes, que desde su nacimiento, número $1 \mathrm{sep}$ tiembre-diciembre 2012 hasta el mes de diciembre de 2019 fue dirigida por el Dr. Gustavo Martínez Mier, quien realizó un trabajo extraordinario en el desarrollo, la publicación y la difusión de la revista. La Sociedad Mexicana de Trasplantes agradece al Dr. Gustavo Martínez Mier su gran esfuerzo y apoyo para la continuidad de la revista.

Por otro lado, debido a la pandemia causada por COVID-19, y en consecuencia el confinamiento y el aislamiento de la mayor parte de la población, se decidió (por razones obligadas) retrasar un mes la publicación impresa de la revista. Además, se continuará con un formato semejante, pero se agregarán algunos segmentos que enriquecerán los contenidos de la revista. El proyecto es ambicioso, pero trataremos de cumplir con lo informado. Se incluirán artículos de revisión que se aceptarán previa invitación de la revista a un experto en el área seleccionada. Se incorporarán artículos dirigidos al Área de Inmunología Básica para actualizar los conceptos de la respuesta inmune, mecanismos de rechazo y la base teórica de la inmunosupresión. Se incluirán también aspectos técnicos de las diferentes cirugías de trasplante incluyendo la sección de "¿Cómo lo hago yo?". Además, está planeado que se puedan publicar uno o dos suplementos anuales dirigidos a un área específica, independientemente de los resúmenes del Congreso Nacional de Trasplantes. Se incluyeron dos segmentos nuevos, siendo el primero de Coordinación y Donación y el segundo en Enfermería de Trasplantes, por lo que ahora, todos aquellos interesados podrán enviar sus trabajos para publicar. Asimismo, se continuará la publicación en ambos formatos que son: en línea (con el portal de la revista) y en publicación impresa.

Por otra parte, se hicieron algunos cambios en los Comités Editoriales y se agregaron los dos segmentos ya mencionados previamente. Se anexaron para el trabajo de coedición a la Dra. Josefina Alberú y a la Dra. Carmen Gracida, quienes (junto a su servidor) trataremos de llevar a buen término el trabajo anteriormente esbozado.

Por último, lo más importante es que tengamos un mejor foro para la publicación de trabajos originales y de investigación de los trasplantes en México. Estamos interesados que los países latinoamericanos y el resto de los países del Continente Americano envíen artículos para publicar en la Revista Mexicana de Trasplantes, para así enriquecer aún más los contenidos. Invitamos a investigadores básicos, clínicos y quirúrgicos a sumarse al esfuerzo de publicar la experiencia de todos ustedes que seguro beneficiará, no sólo a la Revista Mexicana de Trasplantes, sino a toda la comunidad científica en el Continente Americano.

Bienvenidos sean todos ustedes a la Revista Mexicana de Trasplantes.

Correspondencia:

Dr. Federico Javier Juárez-de la Cruz

E-mail: fjjuarez2012@gmail.com 